\title{
Impact of Different Extraction Solvents on Bioactive Compounds and Antioxidant Capacity from the Root of Salacia chinensis $\mathrm{L}$.
}

\author{
Thanh Van Ngo, ${ }^{1,2}$ Christopher James Scarlett, ${ }^{1}$ Michael Christian Bowyer, ${ }^{1}$ \\ Phuong Duc Ngo, ${ }^{3}$ and Quan Van Vuong ${ }^{1}$ \\ ${ }^{1}$ School of Environmental and Life Sciences, The University of Newcastle, P.O. Box 127, Ourimbah, NSW 2258, Australia \\ ${ }^{2}$ Vietnam National University of Forestry, Xuan Mai, Chuong My, Hanoi, Vietnam \\ ${ }^{3}$ Investment and Development of Southern Herb JSC, Quynh Luu District, Nghe An Province, Vietnam
}

Correspondence should be addressed to Thanh Van Ngo; thanh.ngovan@uon.edu.au

and Quan Van Vuong; vanquan.vuong@newcastle.edu.au

Received 29 July 2016; Revised 15 September 2016; Accepted 4 October 2016; Published 11 January 2017

Academic Editor: Eduardo Puértolas

Copyright (C) 2017 Thanh Van Ngo et al. This is an open access article distributed under the Creative Commons Attribution License, which permits unrestricted use, distribution, and reproduction in any medium, provided the original work is properly cited.

\begin{abstract}
This study aimed to study the impact of selected common organic solvents on extractable solids, phytochemical composition, and antioxidant capacity of $S$. chinensis. The results showed that the tested solvents played an important role in extraction of total solid and phytochemical composition as well as antioxidant capacity of S. chinensis. Acetone (50\% v/v) was found to be the optimal extraction solvent for extractable solids (12.2\%), phenolic compounds (60 mg GAE/g DW), flavonoids (100 mg CE/g DW), proanthocyanidins (47.4 mg CE/g DW), and saponins (754 mg EE/g DW) as well as antioxidant capacity (ABTS $334 \mathrm{mM} \mathrm{TE} / \mathrm{g} \mathrm{DW}$, DPPH $470 \mathrm{mM}$ TE/g DW, FRAP $347 \mathrm{mM}$ TE/g DW, and CUPRAC $310 \mathrm{mM}$ TE/g DW). The extract prepared from 50\% acetone had high levels of bioactive compounds (TPC $555 \mathrm{mg}$ GAE/g CRE, flavonoids $819 \mathrm{mg}$ CE/g CRE, proanthocyanidins $392 \mathrm{mg}$ CE/g CRE, and saponins 1,880 mg EE/g CRE) as well as antioxidant capacity (ABTS $414 \mathrm{mM} \mathrm{TE} / \mathrm{g}$, DPPH $407 \mathrm{mM} \mathrm{TE} / \mathrm{g}$, FRAP $320 \mathrm{mg}$ $\mathrm{TE} / \mathrm{g}$, and CUPRAC $623 \mathrm{mM} \mathrm{TE} / \mathrm{g}$ ), thus further confirming that $50 \%$ acetone is the solvent of choice. Therefore, $50 \%$ acetone is recommended for extraction of phenolic compounds, their secondary metabolites, saponins, and antioxidant capacity from the root of S. chinensis for further isolation and utilisation.
\end{abstract}

\section{Introduction}

Salacia chinensis L. (S. chinensis) belongs to the genus Salacia of the family Celastraceae. The $S$. chinensis tree can grow up to 3-10 $\mathrm{m}$ in height and $16 \mathrm{~cm}$ in diameter in the tropical forests of Africa, China, India, and South East Asia including Laos, Cambodia, and Vietnam. S. chinensis has been widely used as a traditional medicine to treat various ailments such as arthritis, leucorrhoea, inflammation, fever, as an astringent, and amenorrhea [1]. In Vietnam, the root of $S$. chinensis has been used for the treatment of rheumatism, back-pain, and debility [2]. S. chinensis has been linked with antimicrobial, antidiabetic, antioxidant, antimutagenic, and anticancer properties due to the material being found to contain high levels of phenolic and flavonoid compounds and possessing strong antioxidant capacity [1].
One of the most important factors affecting the extraction efficiency of bioactive compounds from plant materials and their consequent health benefits is the extraction solvent. Traditionally, S. chinensis has been brewed or decocted in water for use as a traditional medicine in some Asian countries, such as India, Sri Lanka, and Vietnam [2, 3]. Previous studies have used methanol, petroleum ether, chloroform, ethanol, acetone, and water as the solvents for extracting bioactive compounds from $S$. chinensis for further analysis [4-6]. Although extraction solvents have been extensively studied in other plant materials, such as macadamia skin waste [7], S. chinensis fruit pulp [8], and basil leaf [9], none of the previous studies have compared the impact of different common solvents on the extraction efficiency of phenolic compounds from the $S$. chinensis root. Therefore, this study aimed to determine the impact of different common solvents 
(water, absolute methanol, ethanol, acetone, 50\% methanol, $50 \%$ ethanol, and $50 \%$ acetone) on the extraction efficiency of bioactive compounds, as well as antioxidant capacity from the root of $S$. chinensis, in order to identify the most appropriate solvent for further extraction and isolation of bioactive compounds and antioxidant capacity from S. chinensis.

\section{Materials and Methods}

2.1. Materials. The root of S. chinensis L. was collected from Nghe An Province (Vietnam) in May 2015. After collection, the root was sun-dried, which is the traditional preparation method to obtain the dried sample. The dried root was then ground into small particles using a commercial blender (John Morris Scientific, Chatswood, NSW, Australia) and then sieved using a steel mesh sieve (1.4 mm EFL 2000; Endecotts Ltd., London, England). The ground root was kept at $-20^{\circ} \mathrm{C}$ for further analysis.

\subsection{Methods for Characterisation of the Root of S. chinensis}

2.2.1. Extraction Process. Seven common solvents were used for the extraction of bioactive compounds from the ground root of S. chinensis L. including water, absolute methanol, ethanol, acetone (the polarity indexes are 10.2, 5.1, 4.3, and 5.1, resp.), 50\% methanol, 50\% ethanol, and 50\% acetone. The sample was extracted in these solvents by firstly adding $1 \mathrm{~g}$ of sample into $100 \mathrm{~mL}$ of solvent. The mixture was then put in an ultrasonic bath (Soniclean, $220 \mathrm{~V}, 50 \mathrm{~Hz}$ and 250 W; Soniclean Pty Ltd., Thebarton, Australia) with preset conditions: temperature of $35^{\circ} \mathrm{C}$, time of $30 \mathrm{~min}$, and power of $150 \mathrm{~W}$ (the mixture was vortexed thoroughly once every five minutes). Next, the extract was immediately cooled on ice to room temperature and then filtered using filter paper (Whatman, $11 \mu \mathrm{m}$ pore size). Subsequently, the extract was stored in the dark at $-18^{\circ} \mathrm{C}$ for further determination of the extractable solids, total phenols content (TPC), total flavonoids content (TFC), total proanthocyanidins content (TPrC), total saponins content (TSC), and antioxidant capacity (DPPH, FRAP, CUPRAC, and ABTS assays).

\subsubsection{Preparation of Saponin and Phenolic Enriched Extract} from S. chinensis. Firstly, $100 \mathrm{~g}$ of sample was added into $2 \mathrm{~L}$ of $50 \%$ acetone. The mixture was then put in an ultrasonic bath (Soniclean, $220 \mathrm{~V}, 50 \mathrm{~Hz}$ and $250 \mathrm{~W}$, Soniclean Pty Ltd., Thebarton, Australia) with preset condition: temperature of $35^{\circ} \mathrm{C}$, time of $30 \mathrm{~min}$, and power of $150 \mathrm{~W}$ (the mixture was vortexed thoroughly once every five minutes). Next, the extract was immediately cooled on ice to room temperature and then filtered using filter paper (Whatman, $11 \mu \mathrm{m}$ pore size). Subsequently, the extract was condensed to the volume of $150 \mathrm{~mL}$ using a rotary evaporator (Buchi Rotavapor B-480, Buchi Australia, Noble Park, Victoria, Australia) and then freeze-dried to yield the crude extract. This crude extract was stored in dark containers at $-20^{\circ} \mathrm{C}$ for further analysis, including TPC, TFC, TPrC, TSC, and antioxidant capacities (DPPH, FRAP, CUPRAC, and ABTS assays).

2.2.3. Determination of Extractable Solids. Extractable solids were determined according to a method described previously with minor modification [10]. $3 \mathrm{~mL}$ of the extract was put in a pottery tray and then placed in an oven set at $120^{\circ} \mathrm{C}$ for drying during $5 \mathrm{~h}$ to remove all moisture. Extractable solids (ES) were calculated by the following formula:

$$
\mathrm{ES}(\%)=\frac{W \times 100}{3}
$$

( $W$ is weight of $3 \mathrm{~mL}$ of the extraction after drying, in grams).

\subsubsection{Determination of Chemical Properties}

Total Phenolic Content (TPC). TPC of Salacia chinensis root was determined as previously described by [11]. $2.5 \mathrm{~mL}$ of $10 \%(\mathrm{v} / \mathrm{v})$ Folin-Ciocalteu reagent was mixed with $0.5 \mathrm{~mL}$ of diluted sample. The solution was then added to $2 \mathrm{~mL}$ of $7.5 \%$ (w/v) $\mathrm{Na}_{2} \mathrm{CO}_{3}$, followed by thorough mixing and incubating in the dark at room temperature for $1 \mathrm{~h}$. The absorbance at $760 \mathrm{~nm}$ was taken using a UV spectrophotometer (Varian Australia Pty. Ltd., Victoria, Australia); a reagent blank was set at base level (zero). Gallic acid was used as the standard for a calibration curve and the results were expressed as mg of gallic acid equivalents per $g$ of sample dry weight (mg GAE/g DW).

Total Flavonoids Content (TFC). TFC of Salacia chinensis root was determined as previously described by Dailey and Vuong [12]. $2 \mathrm{~mL}$ of deionized water was mixed with $0.15 \mathrm{~mL}$ of $5 \%$ (w/v) $\mathrm{NaNO}_{2}$ and $0.5 \mathrm{~mL}$ of diluted sample. The solution was mixed thoroughly and then left at room temperature for $6 \mathrm{~min}$. Subsequently, $0.15 \mathrm{~mL}$ of $10 \%$ (w/v) $\mathrm{AlCl}_{3}$ was added and the solution was mixed well and allowed to stand for $6 \mathrm{~min}$. Finally, $2 \mathrm{~mL}$ of $4 \%(\mathrm{w} / \mathrm{v}) \mathrm{NaOH}$ and $0.7 \mathrm{~mL}$ of deionized water were added to get the final volume of $5.5 \mathrm{~mL}$. The solution was then mixed thoroughly and allowed to stand for $15 \mathrm{~min}$ at room temperature. The absorbance at $510 \mathrm{~nm}$ was taken using a UV spectrophotometer (Varian Australia Pty. Ltd., Victoria, Australia); a reagent blank was set at base level (zero). Catechin was used as the standard for a calibration curve and the results were expressed as $\mathrm{mg}$ of catechin equivalents per gram of sample dry weight (mg CE/g DW).

Total Proanthocyanidins Content (TPrC). TPrC of Salacia chinensis root was measured as previously described by Dailey and Vuong [12]. $0.5 \mathrm{~mL}$ of diluted sample was mixed with $3 \mathrm{~mL}$ of $4 \%$ vanillin and followed by adding $1.5 \mathrm{~mL} \mathrm{HCl}$ $37 \%$. The solution was mixed and allowed to stand for $15 \mathrm{~min}$. The absorbance was measured using a spectrophotometer at $500 \mathrm{~nm}$; a reagent blank was set at base level (zero). Catechin was used as the standard for a calibration curve and the results were expressed as $\mathrm{mg}$ of catechin equivalents per gram of sample dry weight (mg CE/g DW).

Total Saponin Content (TSC). TSC of Salacia chinensis root was determined as previously described by Vuong et al. [11]. $0.5 \mathrm{~mL}$ of diluted sample was mixed with $0.5 \mathrm{~mL}$ of $8 \%$ vanillin, followed by adding $5 \mathrm{~mL} \mathrm{H}_{2} \mathrm{SO}_{4}(72 \%)$. The solution was mixed thoroughly and placed on ice to cool. The mixture was then incubated in a water bath at $60^{\circ} \mathrm{C}$ for $15 \mathrm{~min}$. The 
mixture was then cooled on ice for approximately $10 \mathrm{~min}$ and the absorbance was then measured at $560 \mathrm{~nm}$; a reagent blank was set at base level (zero). Escin was used as the standard for a calibration curve and the results were expressed as $\mathrm{mg}$ of escin equivalents per gram of sample dry weight (mg EE/g DW).

\subsubsection{HPLC Analysis of Bioactive Components in the Crude} Extract of $S$. chinensis Root. The solution made of $0.02 \mathrm{~g}$ of crude extract of $S$. chinensis root diluted in $2 \mathrm{~mL}$ of $50 \%$ acetone was filtered using a $0.45 \mu \mathrm{m}$ Phenex Syringe filter (Phenomenex). The bioactive components were then measured using a Shimadzu HPLC system (Shimadzu Australia, Rydalmere, NSW, Australia) using UV detection at $254 \mathrm{~nm}$, on a $250 \mathrm{~mm} \times 4.6 \mathrm{~mm}$ Prodigy $5 \mu \mathrm{m}$ ODS3-100A reversed-phase column (Phenomenex Australia Pty. Ltd., Lane Cove, NSW, Australia) which was maintained at $35^{\circ} \mathrm{C}$. The mobile phases consisted of solvent systems A and B; solvent $A$ was deionized water:acetonitrile:orthophosphoric acid, 96.8:3: $0.2(\mathrm{v} / \mathrm{v} / \mathrm{v})$; solvent B was $100 \%$ acetonitrile.

A gradient elution schedule was used as follows: 100\% A from 0 to $10 \mathrm{~min}$; a linear gradient from $100 \% \mathrm{~A}$ to $90 \% \mathrm{~A}$ from 10 to $15 \mathrm{~min}$ and remaining at $90 \%$ A to $25 \mathrm{~min}$; from $90 \%$ A to $85 \%$ A from 25 to $40 \mathrm{~min}$; from $85 \%$ A to $10 \% \mathrm{~A}$ from $40 \mathrm{~min}$ to $42 \mathrm{~min}$; $10 \%$ A to $0 \%$ A from 42 to $52 \mathrm{~min}$ and remaining at $0 \% \mathrm{~A}$ to $57 \mathrm{~min}$ and then back to $100 \% \mathrm{~A}$ at $60 \mathrm{~min}$ with a postrun reequilibration time of $15 \mathrm{~min}$ with $100 \%$ A before the next injection. The injection volume was $50 \mu \mathrm{L}$ of the crude extract solution onto the HPLC and the flow rate was $1 \mathrm{~mL} / \mathrm{min}$.

2.2.6. Determination of Antioxidant Properties. To obtain a greater understanding on the antioxidant properties of the Salacia chinensis root, four antioxidant assays were employed including the ABTS (2,2' - azino-bis(3-ethylbenzthiazoline-6sulphonic acid)) assay, the DPPH (2,2-diphenyl-1-picrylhydrazyl) assay, the CUPRAC (cupric reducing antioxidant capacity) assay, and the FRAP (ferric reducing antioxidant power) assay.

ABTS Assay. ABTS assay described by Thaipong et al. [13] was applied with some modifications. A stock solution was prepared by adding $10 \mathrm{~mL}$ of $7.4 \mathrm{mM}$ ABTS solution to $10 \mathrm{~mL}$ of $2.6 \mathrm{mM} \mathrm{K}_{2} \mathrm{~S}_{2} \mathrm{O}_{8}$ and left at room temperature in the dark for $15 \mathrm{~h}$ and then stored at $-20^{\circ} \mathrm{C}$ until required. The working solution was freshly prepared by diluting $1 \mathrm{~mL}$ of stock solution with approximately $60 \mathrm{~mL}$ of methanol to obtain an absorbance value of $1.1 \pm 0.02$ at $734 \mathrm{~nm}$ at the day of analysis. $2.85 \mathrm{~mL}$ of the working solution was added to $0.15 \mathrm{~mL}$ of diluted sample and left in the dark at room temperature for $2 \mathrm{~h}$ before its absorbance was read at $734 \mathrm{~nm}$ using a UV spectrophotometer (Varian Australia Pty. Ltd., Victoria, Australia). Trolox was used as a standard and the results were expressed as mg trolox equivalents per gram of dry weight (mg TE/g dw).

DPPH Assay. DPPH assay introduced by Thaipong et al. [13] was applied with some modifications. A stock solution was prepared by dissolving $24 \mathrm{mg} \mathrm{DPPH}$ in $100 \mathrm{~mL}$ methanol and then stored at $-20^{\circ} \mathrm{C}$ until required. The working solution was then prepared fresh by mixing $10 \mathrm{~mL}$ stock solution with approximately $45 \mathrm{~mL}$ methanol to obtain an absorbance of $1.1 \pm 0.02$ at $515 \mathrm{~nm} .2 .85 \mathrm{~mL}$ of working solution was added to $0.15 \mathrm{~mL}$ of diluted sample and then left under darkness at room temperature for $3 \mathrm{~h}$ before measuring the absorbance at $515 \mathrm{~nm}$ using a UV spectrophotometer (Varian Australia Pty. Ltd., Victoria, Australia). Trolox was used as standard for a calibration curve and the results were expressed as $\mathrm{mg}$ of trolox equivalents per $\mathrm{g}$ of dry weight (mg TE/g dw).

CUPRAC Assay. CUPRAC assay described by Apak et al. [14] was employed with some modifications. $1 \mathrm{~mL}$ of $\mathrm{CuCl}_{2}$ was mixed with $1 \mathrm{~mL}$ of neocuproine and $1 \mathrm{~mL}$ of $\mathrm{NH}_{4} \mathrm{Ac}$ and $1.1 \mathrm{~mL}$ of diluted sample. The sample was mixed well and incubated at room temperature for $1.5 \mathrm{~h}$ before measuring the absorbance at $450 \mathrm{~nm}$ using a UV spectrophotometer (Varian Australia Pty. Ltd., Victoria, Australia). Trolox was used as standard for a calibration curve and the results were expressed as mg of trolox equivalents per $\mathrm{g}$ of sample (mg $\mathrm{TE} / \mathrm{g} \mathrm{dw}$ ).

FRAP Assay. FRAP assay described by Thaipong et al. [13] was employed with some modifications. A working FRAP solution was prepared by mixing $300 \mathrm{mM}$ Acetate buffer, $10 \mathrm{mM}$ tripyridyl-s-triazine (TPTZ) in $40 \mathrm{mM} \mathrm{HCl}$, and $20 \mathrm{mM}$ $\mathrm{FeCl}_{3}$ in the ratio of $10: 1: 1$ and mixed at $37^{\circ} \mathrm{C}$ in a water bath (Ratek Instruments Pty. Ltd., Victoria, Australia) before use. $2.85 \mathrm{~mL}$ of the working FRAP solution was added to $0.15 \mathrm{~mL}$ of diluted sample and incubated at room temperature in the dark for $30 \mathrm{~min}$ before its absorbance was read at $593 \mathrm{~nm}$ using a UV spectrophotometer (Varian Australia Pty. Ltd., Victoria, Australia). Trolox was used as a standard and the results were expressed as mg trolox equivalents per gram of dry weight (mg TE/g dw).

2.3. Statistical Analysis. The one-way analysis of variance (ANOVA) and the Least Significance Difference (LSD) were conducted using the IBM SPSS statistical software version 23. Data were reported as averages \pm standard deviations. Differences between the mean levels of the components in the different experiments were taken to be statistically significant at $p<0.05$. The Pearson correlation test was employed to determine the correlation coefficients among bioactive compounds and different antioxidant assays.

\section{Results and Discussion}

3.1. Impact of Extraction Solvents on Extractable Solids. The result of this study showed that different solvents had significant effects on the extractable solids yield of $S$. chinensis root (Figure 1). Absolute methanol had the highest extractable solids (15.6\%), followed by $50 \%$ ethanol, $50 \%$ methanol, and $50 \%$ acetone $(14.3 \%, 12.3 \%$, and $12.2 \%$, resp.). Water extracted half of extractable solids in comparison with absolute methanol, whereas absolute ethanol and absolute acetone only extracted $\sim 25 \%$ of extractable solids extracted by absolute methanol. These findings indicated that recovery yields of crude powder extract prepared from S. chinensis 


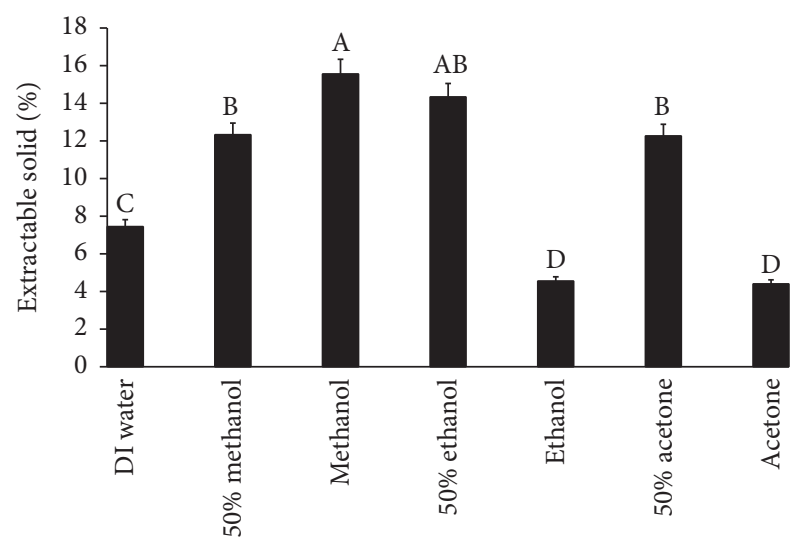

FIGURE 1: Effect of solvents on extractable solids from the root of $S$. chinensis. The values are the mean average of three replications for each solvent \pm standard deviation. Columns not sharing the same superscript letter are significantly different $p<0.05$ (DI water: deionized water).

root could be significantly affected by the extraction solvents. These findings were in agreement with previous studies on Limnophila aromatica [15] and Phoenix dactylifera L. [16], whereby the variation can be explained by the difference in solubility of different compounds in the sample. In general, these findings suggested that absolute methanol or mixture of $50 \%(\mathrm{v} / \mathrm{v})$ water with methanol, ethanol, or acetone was the solvents of choice for yielding high levels of extractable solids.

\subsection{Impact of Extraction Solvents on Total Phenolic Content} (TPC). Our data showed that extraction solvents had a significant impact on the extraction yields of TPC from the root of S. chinensis (Figure 2). The mixture of 50\% (v/v) water with methanol, ethanol, and acetone had the highest extraction yields of TPC, followed by absolute methanol and absolute ethanol, which accounted for approximately $66 \%$ and $43 \%$ of the TPC extracted by $50 \%$ acetone. Water extracted approximately $30 \%$ of TPC in comparison to $50 \%$ acetone, whereas absolute acetone only extracted about $12 \%$ of the TPC extracted by $50 \%$ acetone. These findings further confirmed that extraction solvents play an important role in the extraction of phenolic compounds from the sample, and the mixture of $50 \%(\mathrm{v} / \mathrm{v})$ water with methanol, ethanol, and acetone is the best solvents for maximum extraction of TPC.

These findings were supported by previous studies, which also found that different extraction solvents significantly affected the extraction yields of TPC $[6,7,15,17]$. However, the extraction yields of TPC were different depending on the types of solvent used. For example, Chavan et al. [6] reported that methanol was the best extraction solvent for TPC from the fresh fruit pulp of S. chinensis, while Dailey and Vuong [7] reported that 50\% acetone with water was the best solvent for the extraction of TPC from macadamia skin. Furthermore, Do et al. [15] found that absolute ethanol and acetone were the best extraction solvents for TPC from Limnophila aromatic. The differences can be explained by the variation in polarities of the solvents, which selectively extract different hydrophobic or hydrophilic phenolic compounds in the sample, thus highlighting the importance of investigating and identifying the optimal extraction solvent for each sample type.

\subsection{Impact of Extraction Solvents on Total Flavonoid Content} (TFC). Our study showed that the extraction solvents had a significant effect on the extraction of flavonoids $(p<0.05)$ (Figure 2). The mixture of $50 \%(\mathrm{v} / \mathrm{v})$ water with acetone and ethanol had the highest extraction yields of flavonoids (100 and $89 \mathrm{mg}$ CE/g DW, resp.). This was followed by $50 \%$ methanol ( $85 \mathrm{mg} \mathrm{CE} / \mathrm{g} \mathrm{DW}$ ), while absolute methanol, absolute ethanol, and water could only extract $50 \%, 30 \%$, and $20 \%$ of flavonoids, respectively, in comparison with those of $50 \%$ acetone and $50 \%$ methanol. Absolute acetone extracted the lowest flavonoid levels from S. chinensis. Our findings were supported by previous studies on S. chinensis fruit pulp, Limnophila aromatica, and Macadamia tetraphylla skin waste, which reported that extraction solvents significantly affected flavonoids $[6,7,15]$. The variation can be also explained by the different polarities of compounds which were selectively more soluble in different solvents.

3.4. Impact of Extraction Solvents on Total Proanthocyanidin Content. The current study found that absolute acetone had the highest extraction of proanthocyanidins $(61 \mathrm{mg}$ $\mathrm{CE} / \mathrm{g} \mathrm{DW})$, followed by $50 \%$ acetone (47.4 mg CE/g DW) (Figure 2). 50\% methanol or ethanol only extracted $50 \%$ of proanthocyanidins in comparison to that extracted by absolute acetone. Water was found to extract the lowest content of proanthocyanidins. These findings indicated that extraction solvents play an important role in the extraction efficiency of proanthocyanidins. Water has the highest polarity index, whereas acetone has the lowest polarity index among the tested solvents; thus most proanthocyanidins from S. chinensis are more hydrophilic and thus acetone is the best solvent for extraction of these phenolic compounds.

\subsection{Impact of Extraction Solvents on Total Saponin Content.} The results of this study showed that the best solvent for extraction of saponins was $50 \%$ acetone (754 mg EE/g DW), followed by absolute methanol, ethanol, and $50 \%(\mathrm{v} / \mathrm{v})$ of these solvents with water. The results also revealed that water and absolute acetone had the lowest content of saponins (Figure 2). Previous studies also reported that different extraction solvents significantly affected the extraction efficiency of saponins $[18,19]$. In comparison with some Chinese herbal medicines reported by Chen et al. [20], the total content of saponins in S. chinensis is higher than those of various species of herbs, such as Artemisia capillaries, Codonopsis pilosula, Euryale ferox, and Coix lacryma-jobi.

3.6. Impact of Extraction Solvents on Antioxidant Properties. Four antioxidant assays were used for determining the effect of extraction solvents on the antioxidant capacity of $S$. chinensis extracts. Figure 3 shows that the extraction solvent significantly affected the antioxidant capacity of $S$. chinensis. All four antioxidant assays revealed that antioxidant capacity is in decreasing order with the corresponding solvents used: 

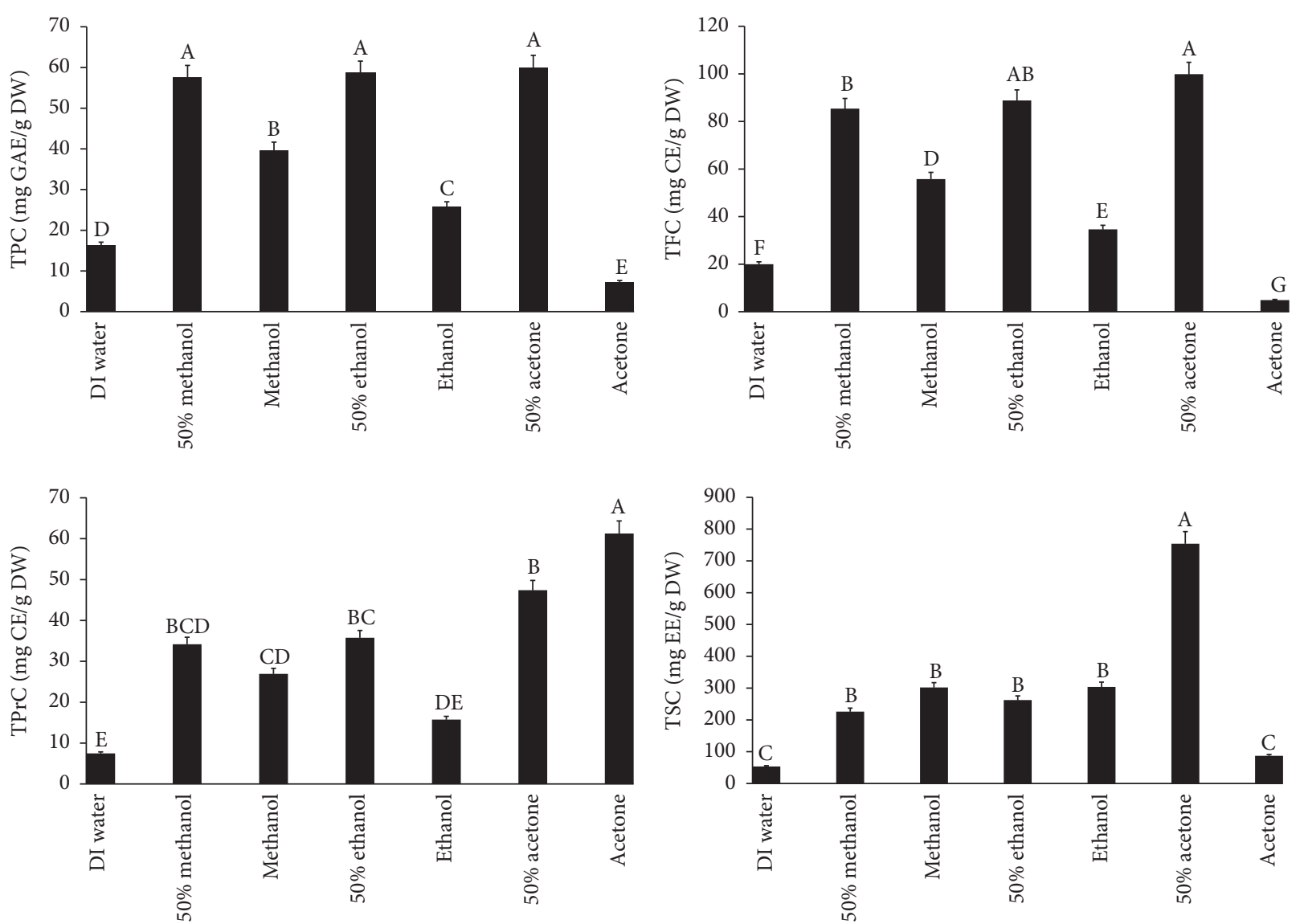

FIGURE 2: Effect of solvents on recovery of total phenolics (TPC), flavonoids (TFC), proanthocyanidins (TPrC), and saponins (TSC) from the root of $S$. chinensis. The values are the mean of three replications for each solvent \pm standard deviation. Columns not sharing the same superscript letter are significantly different $p<0.05$ (DI water: deionized water, GAE: gallic acid equivalent, CE: catechin equivalent, EE: escin equivalent, and DW: dry weight).

$50 \%$ acetone $>50 \%$ ethanol $>50 \%$ ethanol $>$ absolute methanol $>$ absolute ethanol $>$ water $>$ absolute acetone. Previous studies found that extraction solvents significantly affected antioxidant capacities of Saptarangi ( $S$. chinensis L.) fruit pulp [6] and macadamia skin [7]. However, the impact of individual solvents on the antioxidant capacity of different solvents was different. For example, Chavan et al. [6] reported that absolute methanol gave the highest antioxidant capacity, followed by ethanol, acetone, and water that had the lowest antioxidant capacity from Saptarangi (S. chinensis L.) fruit pulp, whereas Dailey and Vuong [7] revealed that the highest antioxidant capacities were seen in the combination of organic solvents (methanol, ethanol, acetonitrile, and acetone) with water in the ratio of $1: 1(\mathrm{v} / \mathrm{v})$.

The differences in impact of solvents on antioxidant capacity of S. chinensis in the current study can be explained by the variation of bioactive groups extracted by the different solvents. Each bioactive group contributed with a different antioxidant power as these groups were found to have differing correlation with antioxidant capacity (Table 2). Table 2 shows that phenolic compounds had a strong correlation with the four antioxidant properties $(r>0.95)$, followed by flavonoids $(r>0.67)$ and saponins $(r>0.6)$. Proanthocyanidins were found to have a weak correlation with antioxidant capacity of $S$. chinensis. These findings revealed that antioxidant capacity of $S$. chinensis was mainly contributed by phenolic compounds, flavonoids, and saponins. These findings were supported by studies on fruit pulp of Salacia chinensis, lilly pilly, and bitter melon, which reported that phenolic compounds, flavonoids, and saponins were mainly responsible for antioxidant activity for these tested materials $[6,21,22]$.

\subsection{Phytochemical and Antioxidant Properties and HPLC} Analysis of the Enriched Extract. As 50\% acetone was found to be the best extraction solvent for both phenolic and saponin compounds from $S$. chinensis root, a crude extract was prepared using $50 \%$ acetone and the results are shown in Table 1 . The results showed that the crude extract had high level of phenolics and saponins as well as potent antioxidant capacity. Compared to the result of previous studies, the TPC, TFC, and TPrC of enriched extract of S. chinensis root (555 mg GAE, $819 \mathrm{mg} \mathrm{CE}$, and $392 \mathrm{mg}$ CE per g crude extract, resp.) are much higher than those of Davidsonia pruriens $\mathrm{F}$. 


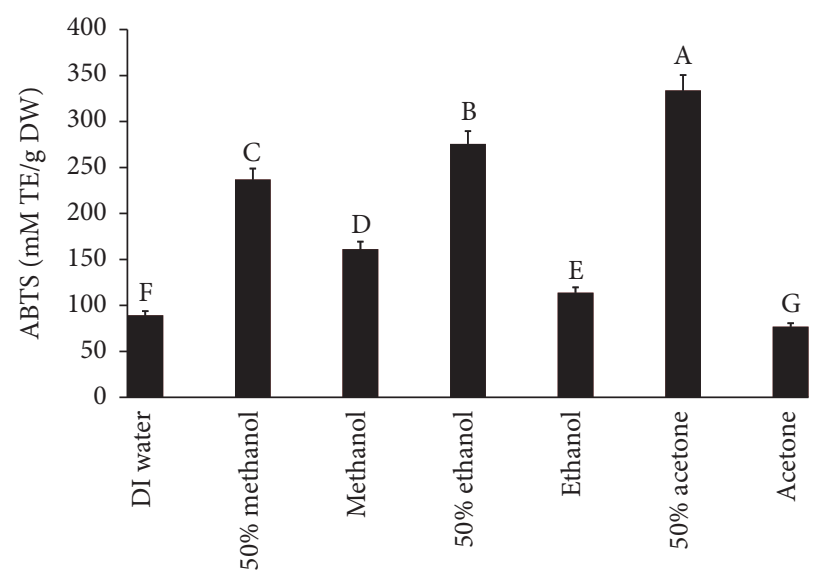

(a)

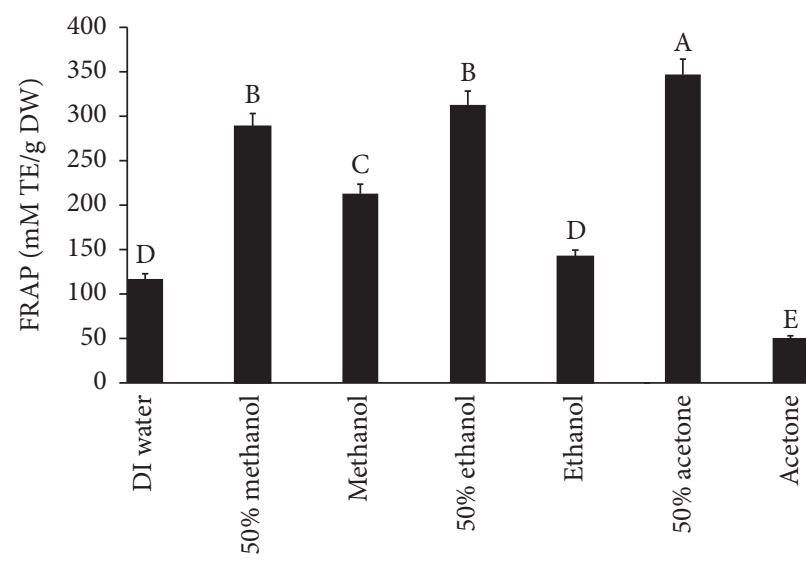

(c)

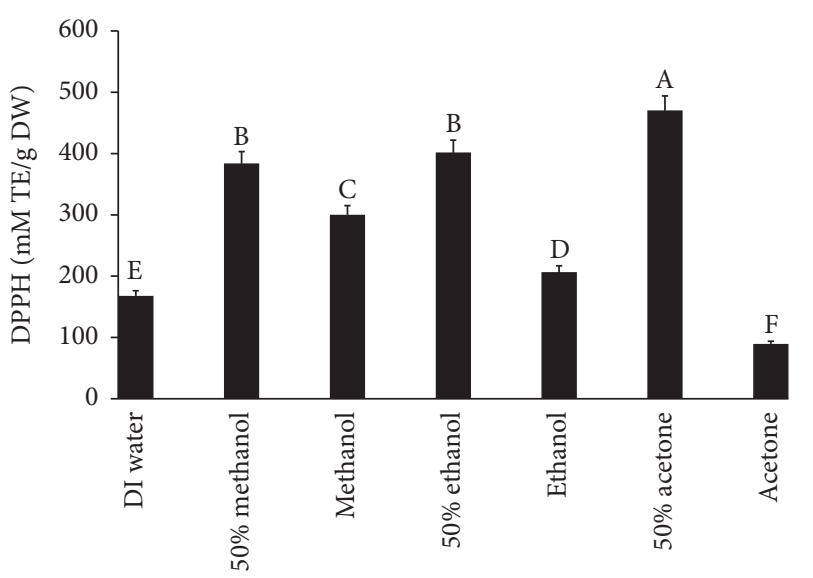

(b)

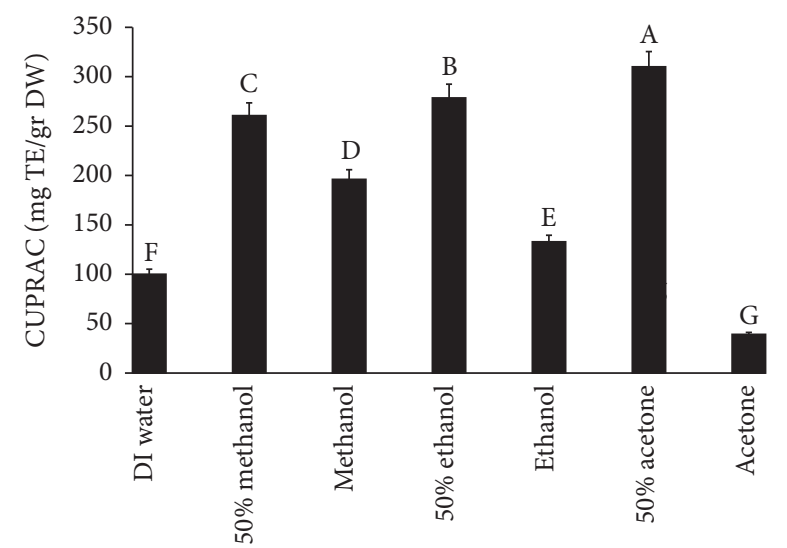

(d)

FIGURE 3: Effect of solvents on antioxidant properties from the root of S. chinensis using various antioxidant assays, including ABTS, DPPH, FRAP, and CUPRAC. The values are the mean of three replications for each solvent \pm standard deviation. Columns not sharing the same superscript letter are significantly different $p<0.05$ (DI water: deionized water).

TABLE 1: Physicochemical and antioxidant properties of the enriched extract prepared by optimal solvent.

\begin{tabular}{lc}
\hline Properties & Values \\
\hline Total phenolic compounds (mg GAE/g CRE) & $555.22 \pm 11.22$ \\
Flavonoids (mg CE/g) & $819.47 \pm 27.06$ \\
Proanthocyanidins (mg CE/g) & $392.09 \pm 2.38$ \\
Saponins (mg EE/g) & $1,880.83 \pm 246.68$ \\
DPPH (mM TE/g) & $407.47 \pm 11.40$ \\
ABTS (mM TE/g) & $414.38 \pm 18.21$ \\
CUPRAC (mM TE/g) & $623.82 \pm 9.77$ \\
FRAP (mM TE/g) & $320.24 \pm 15.70$ \\
\hline
\end{tabular}

Muell (45 mg GAE, $22 \mathrm{mg}$ CE, and $3.2 \mathrm{mg}$ CE per g crude extract, resp.) [23]. Similarly, the saponin content of the $S$. chinensis root enriched extract (1,880 mg EE/g crude extract) is many times higher than that of Carica papaya leaf $(32 \mathrm{mg}$ $\mathrm{EE} / \mathrm{g}$ ) [24]. Therefore, these findings further confirmed that $50 \%$ acetone is the solvent of choice for extraction of bioactive

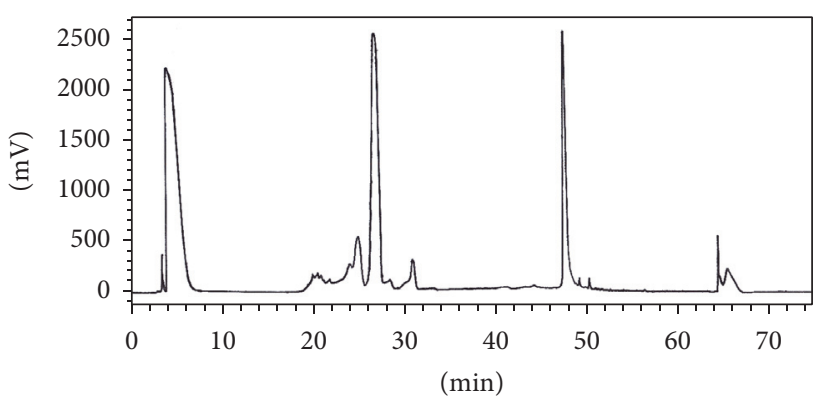

Figure 4: HPLC chromatogram detected at $254 \mathrm{~nm}$ for the crude extract of $S$. chinensis root.

compounds from $S$. chinensis. In addition, these findings also indicated that the crude extract prepared from $50 \%$ acetone has the potential for further testing biological activities such as antimicrobial and anticancer properties.

From the HPLC analysis (Figure 4), there are several peaks in the extract, which can be further isolated for identification as well as testing their properties. 
TABLE 2: Correlation between bioactive compounds and antioxidant properties of the root of S. chinensis.

\begin{tabular}{lcccccccc}
\hline & \multicolumn{2}{c}{ TPC } & \multicolumn{2}{c}{ TFC } & \multicolumn{2}{c}{ TPrC } & \multicolumn{2}{c}{ TSC } \\
& $r$ & $p$ value & $r$ & $p$ value & $r$ & $p$ value & $p$ value \\
\hline ABTS & 0.957 & $* * * *$ & 0.730 & $* * * *$ & 0.322 & ns & 0.664 & $* * *$ \\
FRAP & 0.981 & $* * * *$ & 0.727 & $* * * *$ & 0.202 & ns & 0.606 & $* *$ \\
CUPRAC & 0.989 & $* * * *$ & 0.739 & $* * * *$ & 0.184 & ns & 0.613 & $* *$ \\
DPPH & 0.977 & $* * * *$ & 0.672 & $* * *$ & 0.248 & ns & 0.602 \\
\hline
\end{tabular}

Note: $* * * *$ : extremely significant ( $p$ value $<0.0001), * * *$ : extremely significant $(0.0001<p$ value $<0.001)$, $* *$ : very significant $(0.001<p$ value $<0.01)$, and ns: not significant ( $p$ value $\geq 0.05$ ).

\section{Conclusion}

This study demonstrated that the extraction solvents play an important role in the extraction of important bioactive groups from S. chinensis. Absolute organic solvents or water was not effective, whereas $50 \%$ ethanol and $50 \%$ acetone were solvents of choice for yielding high content of extractable solids, phenolic compounds, and flavonoids. Among these two solvents, $50 \%$ acetone was found to have the highest levels of saponins as well as high antioxidant capacity. This study also prepared phenolic and saponin enriched extracts using $50 \%$ acetone and further confirmed that $50 \%$ acetone was the solvent of choice for yielding high content of phenolics, saponins, and antioxidant properties. Therefore, $50 \%$ of acetone is recommended for extraction of phenolic compounds, their secondary metabolites, and saponins from the root of S. chinensis for further isolation and utilisation.

\section{Additional Points}

Practical Applications. The medicinal properties of herbal plants are mostly determined by the contents of bioactive compounds, such as phenolic compounds, flavonoids, and saponins and the antioxidant capacities of the plants. Salacia chinensis has been used widely for prevention and treatment of various diseases, such as arthritis, diabetes, and obesity, and therefore it is a potential material for further research. In this study, we have optimised the conditions for extraction of bioactive compounds and determined the antioxidant properties in S. chinensis root. The result showed that optimal conditions for extraction of bioactive compounds from S. chinensis root can be applied for further isolation and utilisation in the food and pharmaceutical industries.

\section{Competing Interests}

The authors declare no conflict of interests.

\section{Acknowledgments}

The authors also kindly thank to Faculty of Science and IT, University of Newcastle; the Vietnamese Government through the Vietnam International Education Development (VIED); the Ministry of Education and Training; the Ministry of Agriculture and Rural Development and the University of Newcastle for awarding a VIED-TUIT scholarship to Thanh Van Ngo.

\section{References}

[1] J. J. Chavan, D. M. Ghadage, A. S. Bhoite, and S. D. Umdale, "Micropropagation, molecular profiling and RP-HPLC determination of mangiferin across various regeneration stages of Saptarangi (Salacia chinensis L.)," Industrial Crops and Products, vol. 76, pp. 1123-1132, 2015.

[2] V. C. Vo, Dictionary of Vietnamese Medicinal Plants, Medicinal Publishing House, 1997.

[3] M. H. S. Jayawardena, N. M. W. De Alwis, V. Hettigoda, and D. J. S. Fernando, "A double blind randomised placebo controlled cross over study of a herbal preparation containing Salacia reticulata in the treatment of type 2 diabetes," Journal of Ethnopharmacology, vol. 97, no. 2, pp. 215-218, 2005.

[4] S. S. Periyar, P. M. Balu, S. Kamalraj, V. Raja, and S. Murugesan, "Free radical scavenging activity of Salacia chinensis root extract in streptozotocin-induced diabetic rats," Bioresearch Bulletin, vol. 4, no. 1, 2014.

[5] M. S. Sikarwar and M. B. Patil, "Antihyperlipidemic activity of Salacia chinensis root extracts in triton-induced and atherogenic diet-induced hyperlipidemic rats," Indian Journal of Pharmacology, vol. 44, no. 1, pp. 88-92, 2012.

[6] J. J. Chavan, U. B. Jagtap, N. B. Gaikwad, G. B. Dixit, and V. A. Bapat, "Total phenolics, flavonoids and antioxidant activity of Saptarangi (Salacia chinensis L.) fruit pulp," Journal of Plant Biochemistry and Biotechnology, vol. 22, no. 4, pp. 409-413, 2013.

[7] A. Dailey and Q. V. Vuong, "Effect of extraction solvents on recovery of bioactive compounds and antioxidant properties from macadamia (Macadamia tetraphylla) skin waste," Cogent Food \& Agriculture, vol. 1, no. 1, Article ID 1115646, 2015.

[8] J. J. Chavan, D. M. Ghadage, P. R. Kshirsagar, and S. S. Kudale, "Optimization of extraction techniques and RP-HPLC analysis of antidiabetic and anticancer drug Mangiferin from roots of 'Saptarangi' (Salacia chinensis L.)," Journal of Liquid Chromatography and Related Technologies, vol. 38, no. 9, pp. 963-969, 2015.

[9] U. Złotek, S. Mikulska, M. Nagajek, and M. Świeca, “The effect of different solvents and number of extraction steps on the polyphenol content and antioxidant capacity of basil leaves (Ocimum basilicum L.) extracts," Saudi Journal of Biological Sciences, vol. 23, no. 5, pp. 628-633, 2016.

[10] Q. V. Vuong, J. B. Golding, M. H. Nguyen, and P. D. Roach, "Production of caffeinated and decaffeinated green tea catechin powders from underutilised old tea leaves," Journal of Food Engineering, vol. 110, no. 1, pp. 1-8, 2012.

[11] Q. V. Vuong, S. Hirun, P. D. Roach, M. C. Bowyer, P. A. Phillips, and C. J. Scarlett, "Effect of extraction conditions on total phenolic compounds and antioxidant activities of Carica papaya leaf aqueous extracts," Journal of Herbal Medicine, vol. 3, no. 3, pp. 104-111, 2013. 
[12] A. Dailey and Q. Vuong, "Optimum conditions for microwave assisted extraction for recovery of phenolic compounds and antioxidant capacity from Macadamia (Macadamia tetraphylla) skin waste using water," Processes, vol. 4, no. 1, article 2, 2016.

[13] K. Thaipong, U. Boonprakob, K. Crosby, L. Cisneros-Zevallos, and D. Hawkins Byrne, "Comparison of ABTS, DPPH, FRAP, and ORAC assays for estimating antioxidant activity from guava fruit extracts," Journal of Food Composition and Analysis, vol. 19, no. 6-7, pp. 669-675, 2006.

[14] R. Apak, K. Güçlü, M. Özyürek, and S. E. Karademir, "Novel total antioxidant capacity index for dietary polyphenols and vitamins $\mathrm{C}$ and $\mathrm{E}$, using their cupric ion reducing capability in the presence of neocuproine: CUPRAC method," Journal of Agricultural and Food Chemistry, vol. 52, no. 26, pp. 7970-7981, 2004.

[15] Q. D. Do, A. E. Angkawijaya, P. L. Tran-Nguyen et al., "Effect of extraction solvent on total phenol content, total flavonoid content, and antioxidant activity of Limnophila aromatica," Journal of Food and Drug Analysis, vol. 22, no. 3, pp. 296-302, 2014.

[16] W. Kchaou, F. Abbès, C. Blecker, H. Attia, and S. Besbes, "Effects of extraction solvents on phenolic contents and antioxidant activities of Tunisian date varieties (Phoenix dactylifera L.)," Industrial Crops and Products, vol. 45, pp. 262-269, 2013.

[17] C. T. Sulaiman, K. V. Thushar, S. George, and I. Balachandran, "Phenolic characterisation of selected Salacia species using LCESI-MS/MS analysis," Natural Product Research, vol. 28, no. 13, pp. 1021-1024, 2014.

[18] O. A. Wintola and A. J. Afolayan, "Phytochemical constituents and antioxidant activities of the whole leaf extract of Aloe ferox Mill.," Pharmacognosy Magazine, vol. 7, no. 28, pp. 325-333, 2011.

[19] T. Pasaribu, D. A. Astuti, E. Wina, and A. Sumiatisetiyono, "Saponin content of Sapindus rarak pericarp affected by particle size and type of solvent, its biological activity on Eimeria tenella oocysts," International Journal of Poultry Science, vol. 13, no. 6, pp. 347-352, 2014.

[20] Y.-F. Chen, H.-Y. Roan, C.-K. Lii, Y.-C. Huang, and T.-S. Wang, "Relationship between antioxidant and antiglycation ability of saponins, polyphenols, and polysaccharides in Chinese herbal medicines used to treat diabetes," Journal of Medicinal Plants Research, vol. 5, no. 11, pp. 2322-2331, 2011.

[21] S. Tan, C. Stathopoulos, S. Parks, and P. Roach, "An optimised aqueous extract of phenolic compounds from bitter melon with high antioxidant capacity," Antioxidants, vol. 3, no. 4, pp. 814829, 2014.

[22] Q. V. Vuong, S. Hirun, T. L. K. Chuen et al., "Physicochemical composition, antioxidant and anti-proliferative capacity of a lilly pilly (Syzygium paniculatum) extract," Journal of Herbal Medicine, vol. 4, no. 3, pp. 134-140, 2014.

[23] T. L. K. Chuen, Q. V. Vuong, S. Hirun, M. C. Bowyer, C. D. Goldsmith, and C. J. Scarlett, "Optimum aqueous extraction conditions for preparation of a phenolic-enriched Davidson's plum (Davidsonia pruriens F. Muell) extract," International Journal of Food Science and Technology, vol. 50, no. 11, pp. 24752482, 2015.

[24] Q. V. Vuong, S. Hirun, T. L. K. Chuen et al., "Antioxidant and anticancer capacity of saponin-enriched Carica papaya leaf extracts," International Journal of Food Science and Technology, vol. 50, no. 1, pp. 169-177, 2015. 

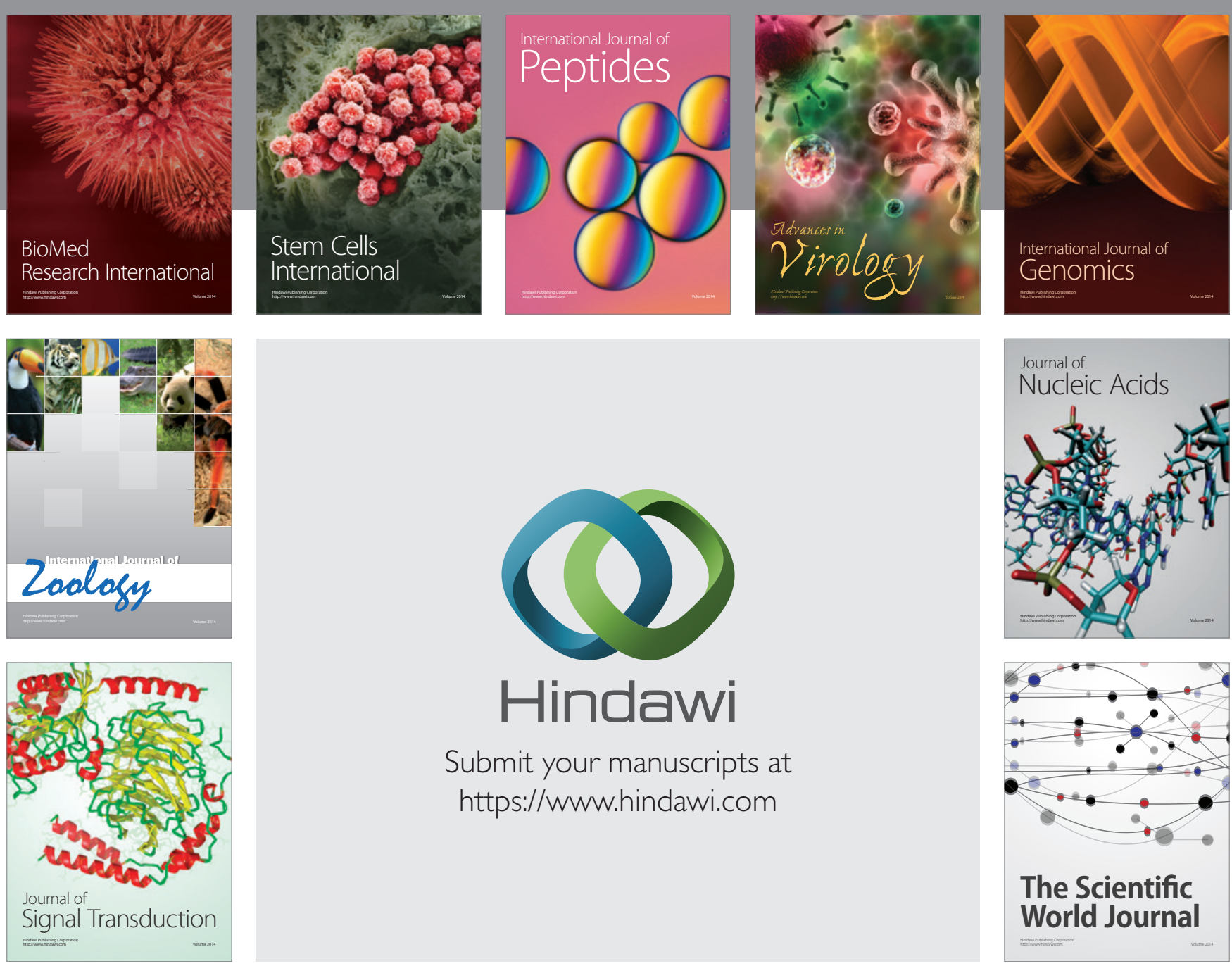

Submit your manuscripts at

https://www.hindawi.com
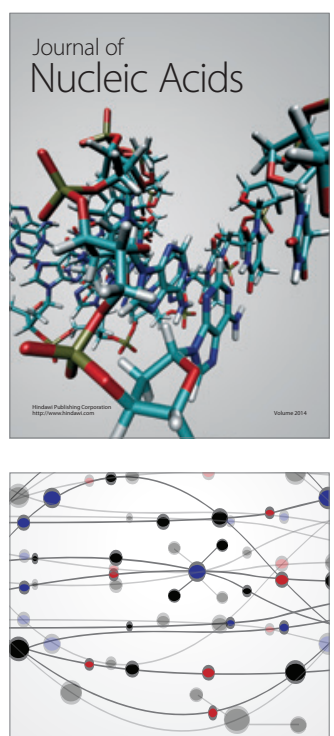

The Scientific World Journal
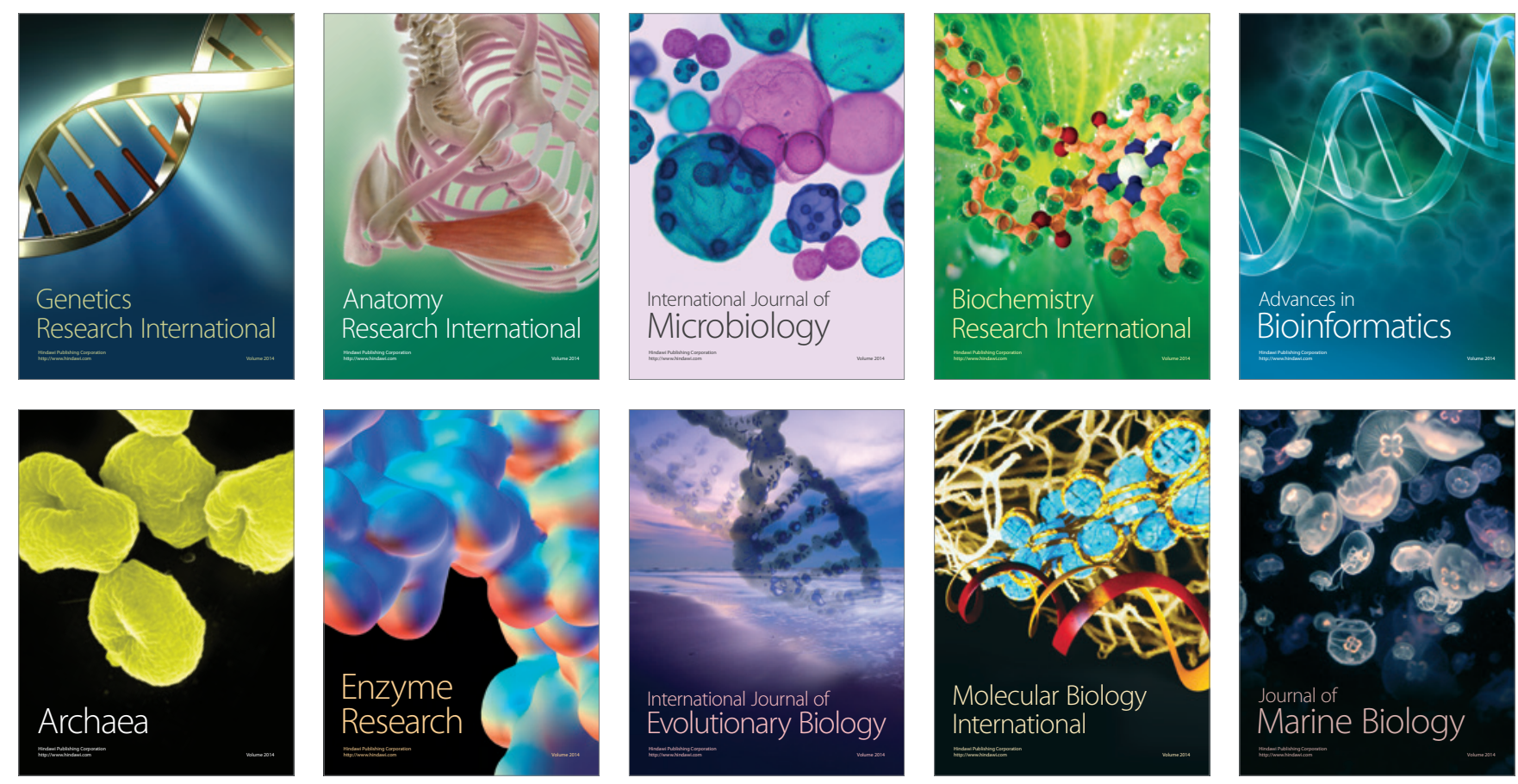